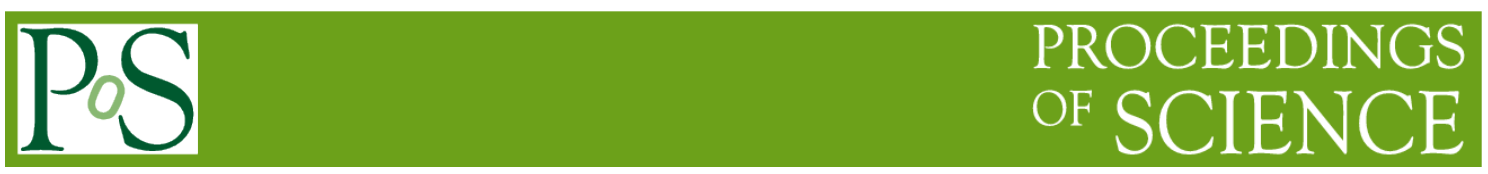

\title{
Link Analysis of VANET in Interchanges of Expressway
}

\author{
Ming-Jer Chiang ${ }^{1}$ \\ Dept. of Electrical Engineering, Chang Gung University, Taoyuan City, 33302, Taiwan(ROC) \\ E-mail: d96210060stmail.cgu.edu.tw
}

\section{Prasan Kumar Sahoo, Shih-Lin Wu}

Dept. of Computer Science and Information Engineering, Chang Gung University, Taoyuan City, 33302, Taiwan(ROC)

E-mail: pksahoo@mail.cgu.edu.tw, slwu@mail.cgu.edu.tw

In recent years, the Vehicular Ad-hoc Networks(VANET) is growing rapidly. There are many applications of the VANET. Especially, the VANET is applied to in the urban-roads and expressways. In this paper, a city is considered as well as the expressway scenario while the analzing the communiation between the street-aware information with vehicles. The length of the vehicles is considered to analyze the communication among cars including those not only waiting at the traffic square but also passing through after obtaining the traffic signal clearly in the interchange. We describe the scenario of interchange of expressway and utilizes the simulation in order to provide useful information. The result of the experiment analysis shows that we simulate average end-to-end delay and packet delivery ratio in the interchange of expressway. We will combine different theories of vehicle in the future work.

ISCC 2015

18-19, December, 2015

Guangzhou, China

${ }^{1}$ Speaker 


\section{Introduction}

The vehicular ad hoc networks(VANET) refers to the communication and connectivity for vehicle. Many researcher utilized different theories to architect the VANET. For example, researchers use the high-speed feature of vehicles in the highway, utilized the density of vehicles, considered the traffic accidents, utilized the geographic routing and exchange useful information. On the orther hands, VANET displays many challenges on communcation, connectivity, technology and protocols that they expand the requirement research in the research field.

In the challenges of VANET, the interchange of expressway is an impartment place for vehicles. The communication among vehicles can be used to realize active safety services of the vehicles and drivers such as weather information, accident warning, update traffic and safe navigation systems. There are many moving cars equipped with the connectivity that the devices are uesed form the accourate devices ad hoc networks. The spots of those networks have virtually unlimited lifetimes and long transmission ranges. If the drivers can get information about the routing statement, they can make a decision whether to change their next route of the destination or not before they are passing through the interchange. In this way, they may save their travel time and reduce fuel consumption.

This paper is to focus on the analysis of interchange of expressway. We will describe the scenario of interchange of expressway and utilize the simulation to provide information in VANET; besides, our propose work used the communication among cars with many velocities on a several lane road with many intersections. Based on the experiment in the packet forwarding, our simulation results show that it is sound to the cars with the least velocity. In any intersection, the duration of communication among cars decreases as to the cars with higher velocity. The information will be referred and utilized by us to combine different theories in research of VANET in the future work.

The organization of the paper show as follows: we describe reevant work in Section II; in Section III, we design our problem formulation. Our experiment evaluation and conclusions are given in Section IV and Section V respectively.

\section{Related Work}

The vehicle connectivity and communication are major research field in VANET. In a research [1], the authors consider the high-velocity scenario of vehicles to guarantee the vehicles' driving safety so that the drivers of vehicles can get the road environment perception information early while passing a road and being prone to accident on highway. In inter-vehicle, the authors [1] also utilize the dynamics model and reminded control approach to give the driving state in the following period.

In a research[2], the authors thought that the communication success had to consider the density of cars. To enhance the warning messages of the dissemination of among cars, the authors [2] use the density of vehicles to make decisions of the importance of predicting in vehicular environments. As a result, the higher density will allow shorter and more reliable wireless links .

In a research[3], the authors pointed out that there were about 0.15 million human being die on the roads in the western world every year according to the UN Economic report of 2011. The authors [3] thought that the use of new sensor and communication technologieswound 
shrink this figure. When the technologies were put into place, cars could be part of a VANET capable of informing the car accidents to the drivers.

The Geographic routing is most investigated research. In a research[4], the authors pointed out that it was for reliable and efficient dissemination of information in VANET. In a research[5], the authors pointed out that we could exchange useful information among vehicles and the roadside infrastructures so that VANET is making an intelligent use of them. There are several possible implementation for this technology, for example, the emergency warning system and adaptive driving control can be adopted to avoid accident among vehicles.

\section{Problem Formulation}

In this proposed work, we consider that the communication link duration analysis among vehicles passing through various types of intersections and freeway interchanges of the city should be studied.

In our previous researches [6], we consider the crossroad intersection as shown in Fig. 1. A two lanes of urban road is taken into account for both crossroad and traffic circle intersection. It is to be mentioned that there are several large crossroad intersections available in big cities with traffic signals and the vehicles from one end are allowed to cross the intersection upon getting the green signal. Upon getting the signal clearance, a vehicle may drive straight through the crossroad intersection or turn left or right. It is assumed here that U-turn be not allowed for the vehicles in the current crossroad scenario. Under such assumption, the vehicle is near the intersection that it must stop at traffic signal during period of time. If the traffic signal is red, the cars can cross through intersection upon getting the signal clearance. In our case, as shown in Fig. 1. The cars travele along Lane A that they can drive through Lane R, Q, or P after passing at the crossroad intersection $\mathrm{T}$.

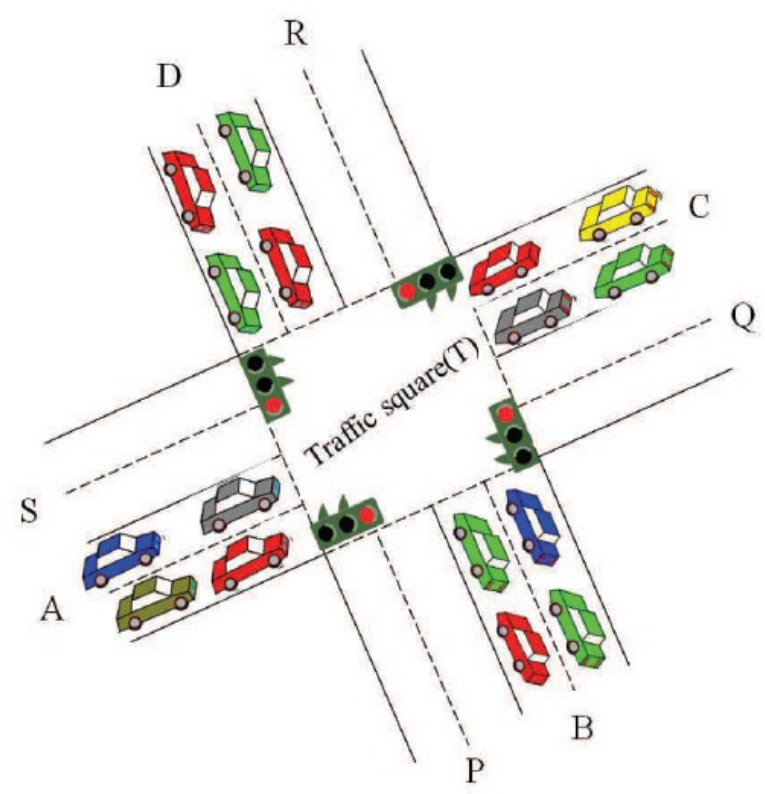

Figure 1: Crossroad Intersection Scenario.

In another scenario, vehicles moving on the expressway and flyover the city roads may carry important messages. Vehicles passing through the interchanges of the expressway may come within communication range of the vehicles passing through the city roads and can form the communication links with them. As shown in Fig. 2, vehicles may exit from the freeway 
through any interchange and go through the traffic clearance before entering the city roads. Hence, it may affect the communication link among the vehicles of the city roads; therefore, it may affect the end-to-end packet forwarding delay. Based on the physical scenario of the city roads and interchanges, we are going to analyze the duration of the communication links between the vehicles when they are passing through any intersection (crossroad or traffic circle) or interchanges of the city. It is assumed that each vehicle should know its location information through GPS and the length of the vehicles may vary. Under these assumption, the following notations are considered for the analysis.

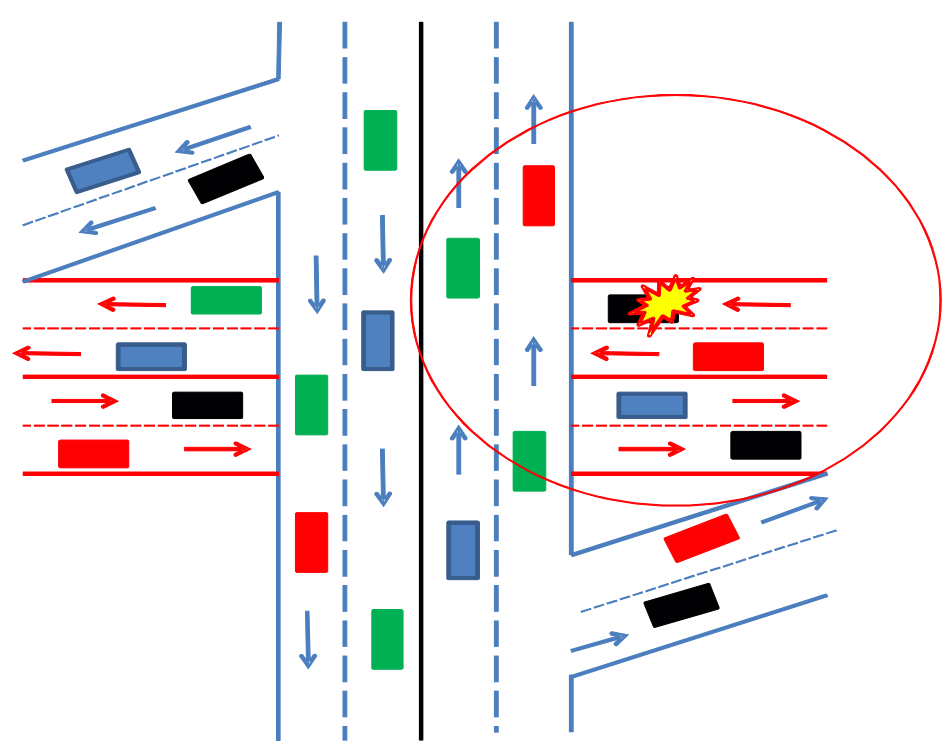

Figure 2: Example of Interchange of an Expressway.

\section{Experiment Evaluation}

In this section, the ways as proposed are evaluated by using VanetMobiSim [7], which is an easy by using simulator and supports the cars mobility to a higher degree of realism which can generate the trace file of route of simulation for our simulation. In addition, we observe over transport layer of the packet level simulation, supporting ad-hoc path protocols, data broadcasting and propagation models. We use NS2 simulator [8] that the tool of mobility of nodes may be definied both directly by using a mobility trace file and the simulation file.

\subsection{Simulation Setup}

The scenario, we set intersection of the area is $1000 \mathrm{~m}^{2}$. In our simulation, the nodes of cars is given to be $20 \mathrm{Km} / \mathrm{h} \sim 60 \mathrm{Km} / \mathrm{h}$ with variable speeds. There are three zones that the velocity of the cars is categorized. The velocity of zone- 1 cars ranges is $20 \mathrm{~km} /$ hour, zone- 2 cars ranges is $40 \mathrm{Km} /$ hour, and zone- 3 cars ranges is $60 \mathrm{Km} /$ hour. The Communication range of each car is given at $100 \mathrm{~m} \sim 250 \mathrm{~m}$. The safe distance between either two cars is given to be $5 \mathrm{~m} \sim 10 \mathrm{~m}$. We use two lengths, $5 \mathrm{~m}$ and $10 \mathrm{~m}$, to be the length of each car. In IEEE 802.11 MAC with two ray ground propagation model and AODC routing, we use NS2 simulator to simulate the average end-to-end packet delay and the packet delivery ratio. 


\subsection{Simulation Result}

In this simulation, we excute the results of the experiment in terms of duration of end-toend packet delivery ratio, for several number of cars changing the lanes.

In our simulation, we consider using the regulate length of car of $5 \mathrm{~m}$. The duration of endto-end packet delivery for different velocity of the cars changing their direction is shown in Fig. 3. It is observed that the velocity is $60 \mathrm{Km} / \mathrm{h}$ and the number of the cars of 60 has higher packet delivery ratio. In the velocity is $60 \mathrm{Km} / \mathrm{h}$ and $80 \mathrm{Km} / \mathrm{h}$ with the number of cars of 80 , the packet delivery ratio is less than the number of cars of 80 becatse of the packet message collision by the node of high density.

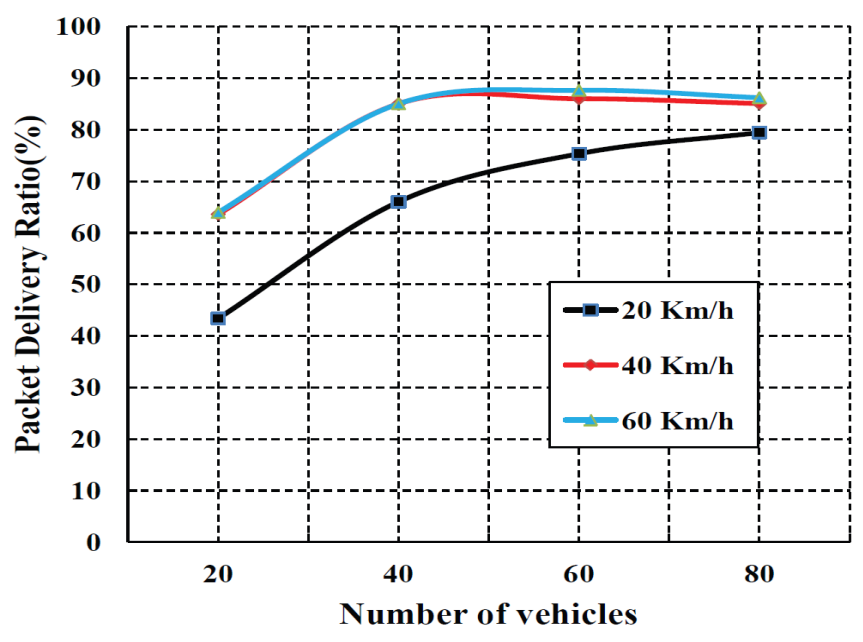

Figure 3: Different Velocitis for Packet Delivery Rtio with the Length $=5 \mathrm{~m}$ of the car.

We also saw the duration of end-to-end packet delivery ratio with the length $=10 \mathrm{~m}$ of the car with change in direction shown in Fig.4. This is observed that the the car with velocity is $60 \mathrm{Km} / \mathrm{h}$ and the number of 60 having high packet delivery ratio.

In our experiment use the AODV Routing, we use the length of car of $5 \mathrm{~m}$ and $10 \mathrm{~m}$. We considered the change in direction. We used the velocity of $60 \mathrm{Km} / \mathrm{h}$ and the number of the cars from 20 to 60. We compared our model with DSR and DSDV.

In Fig. 5 and Fig. 6, we saw our model having higher packet delivery ratio than DSR and DSDV. In the number of the cars is 20 , our model is close to DSR the packet delivery ratio.

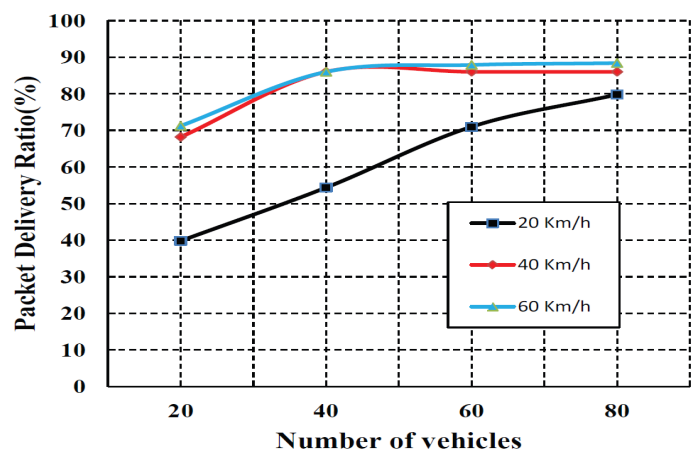

Figure 4: Different Velocitis for Packet Delivery Ratio with the Lngth=10m of the car. 


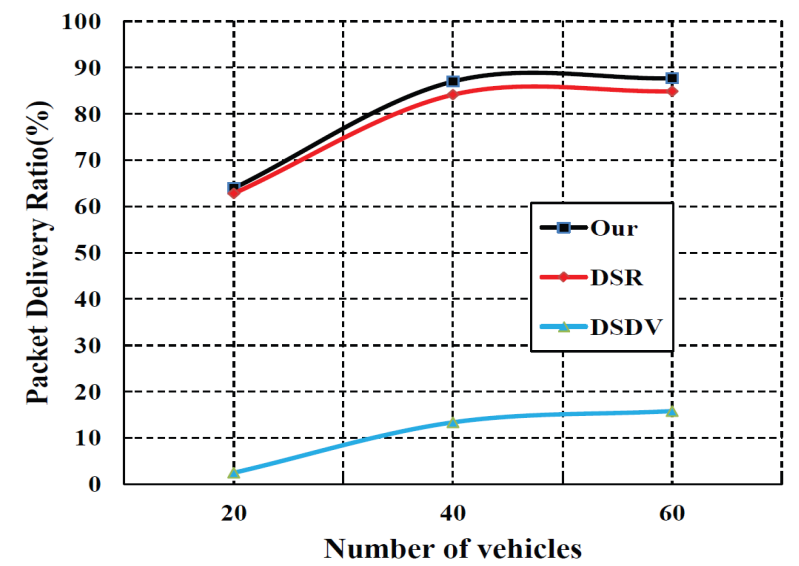

Figure 5: Comparision Packet Delivery Ratio with the Lngth $=5 \mathrm{~m}$ of the car.

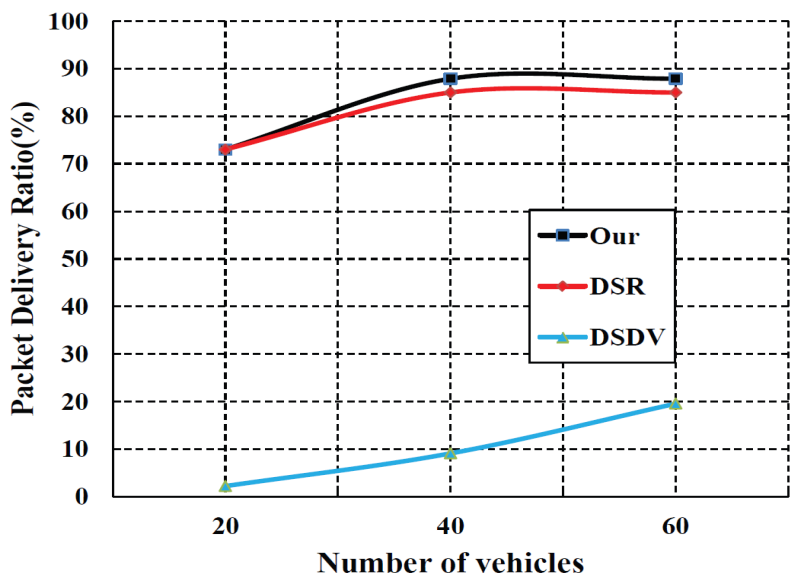

Figure 6: Comparision Packet Delivery Ratio with the Length $=10 \mathrm{~m}$ of the car.

\section{Conclusion}

In this paper, we use the interchange of expressway to design our analysis. We consider several lenghs of vehicles, different numbers of vehicles and different speeds of vehicles. We use speed, density and communication of vehicle between the interchange of expessway to simulate the packet delivery ratio and the average end-to-end delay. The result shows that we can combine the theories of the section of the geographic-based and the density-based commonly. We will use the result of this paper to exam the communication among cars by using the drivers behaviors with other important theories of VANET in our future work.

\section{References}

[1] Xiaofeng Tang, Feng Gao, Guoyan Xu, Nenggen Ding, Yao Cai, Mingming Ma and Jianxing Liu, Sensor Systems for Vehicle Environment Perception in a Highway Intelligent Space System [J], Sensors, Volume 14, Issue 5, 2014, pp. 8513-8527.

[2] Julio A. Sanguesa, Manuel Fogue, Piedad Garrido, Francisco J. Martinez,Juan-Carlos Cano, Carlos T. Calafate and Pietro Manzoni, An Infrastructureless Approach to Estimate Vehicular Density in Urban Environments[J], Sensors, Volume 14, Issue 2, 2013, pp. 2399-2418. 
[3] Gustavo Marfia, Marco Roccetti, Alessandro Amoroso, and Giovanni Pau, Safe Driving in LA: Report from the Greatest Intervehicular Accident Detection Test Eve[J]r, IEEE TRANSACTIONS ON VEHICULAR TECHNOLOGY, VOL. 62, NO. 2, FEBRUARY 2013.pp. 522-535.

[4] Omprakash Kaiwartya, Sushil Kumar, D. K. Lobiyal, Abdul Hanan Abdullah and Ahmed Nazar Hassan, Performance Improvement in Geographic Routing for Vehicular Ad Hoc Network[J]s, Sensors ,Volume14, Issue12, 2014, pp22342-22371.

[5] Juan Antonio Nazabal, Francisco Falcone, Carlos Fernandez-Valdivielso and Ignacio Raul Matias, Development of a Low Mobility IEEE 802.15.4 Compliant VANET System for Urban Environments[J], Sensors, Volume 13, Issue 6, 2013, pp. 7065-7078.

[6] Prasan Kumar Sahoo, M.-J. Chiang and S.-L. Wu, Connectivity Modeling of Vehicular Ad Hoc Networks in Signalized City Roads[C], IEEE ICPP, Taiwan,13-16 September, 2011.

[7] VanetMobiSim Project[Networks], http://vanet.eurecom.fr,Jerome Harri and Marco Fiore, 2006.

[8] NS2 network simulator[Networks], www.isi.edu/nsnam/ns/, 2015 